\title{
USE OF PRIOR INFORMATION FOR BAYESIAN EVALUATION OF BRIDGING STUDIES
}

\author{
Chin-Fu Hsiao, Yu-Yi Hsu, and Hsiao-Hui Tsou
}

Division of Biostatistics and Bioinformatics, National Health Research Institutes Zhunan Town, Miaoli County, Taiwan

\section{Jen-pei Liu}

Division of Biometry, Department of Agronomy, National Taiwan University, Taipei, Taiwan

The ICH E5 guideline defines a bridging study as a supplementary study conducted in the new region to provide pharmacodynamic or clinical data on efficacy, safety, dosage, and dose regimen to allow extrapolation of the foreign clinical data to the population of the new region. Therefore, a bridging study is usually conducted in the new region only after the test product has been approved for commercial marketing in the original region based on its proven efficacy and safety. In this paper we address the issue of analysis of clinical data generated by the bridging study conducted in the new region to evaluate the similarity for extrapolation of the foreign clinical data to the population of the new region. Information on efficacy, safety, dosage, and dose regimen of the original region cannot be concurrently obtained from the local bridging studies but available in the trials conducted in the original region. Liu et al. (2002) have proposed a Bayesian approach to synthesize the data generated by the bridging study and foreign clinical data generated in the original region for assessment of similarity based on superior efficacy of the test product over a placebo control. However, the results of the bridging studies using their approach will be overwhelmingly dominated by the results of the original region due to an imbalance of sample sizes between the regions. Therefore, in this paper we propose a Bayesian approach with the use of a mixture prior for assessment of similarity between the new and original region based on the concept of positive treatment effect. Methods for sample size determination for the bridging study are also proposed. Numerical examples illustrate applications of the proposed procedures in different scenarios.

Key Words: Bayesian approach; Bridging study; Extrapolation; Similarity.

\section{INTRODUCTION}

Recently, geotherapeutics has attracted much attention from sponsors as well as regulatory authorities. However, the key issues lie on when and how to address

Received April 29, 2005; Accepted July 28, 2006

The views expressed in this article are personal opinions of the authors and may not necessarily represent the position of the National Health Research Institutes and National Taiwan University, Taiwan.

Address correspondence to Jen-pei Liu, Division of Biometry, Department of Agronomy, National Taiwan University, 1 Section 4, Roosevelt Road, Taipei, Taiwan; Fax: 886-2-3366-4791; E-mail: jpliu@ntu.edu.tw 
the geographic variations of efficacy and safety for the product development. It will strongly depend upon the size of the market, development cost, and the factors influencing the clinical outcomes for evaluation of efficacy and safety. If the size of the market for some new geographic region is sufficiently large, then it is understandable that the sponsor may be willing to repeat the whole clinical development program after the test product has completed its development plan and maybe obtain the market approval in the original region. Ideally, one, of course, can directly conduct studies in the new region with similar sample size to the phase III trials conducted in the original region for confirmation of the efficacy observed in the original region. Nonetheless, extensive duplication of clinical evaluation in the new region not only demands valuable development resources but also delay availability of the test product to the needed patients in the new regions. To address this issue, the International Conference on Harmonisation $(\mathrm{ICH})$ has published a guideline entitled "Ethnic Factors in the Acceptability of Foreign Clinical Data" known as ICH E5 (1998).

A general framework is provided by the ICH E5 document for evaluation of the impact of ethnic factors on the efficacy, safety, dosage, and dose regimen. The ethnic factors are classified into the following two categories by the ICH E5 guideline. Intrinsic ethnic factors are factors that define and identify the population in the new region and may influence the ability to extrapolate clinical data between regions. They are more genetic and physiologic in nature, e.g., genetic polymorphism, age, gender, etc. On the other hand, extrinsic ethnic factors are factors associated with the environment and culture. Extrinsic ethnic factors are more social and cultural in nature, e.g., medical practice, diet, practices in clinical trials, and conduct. In addition, the ICH E5 guideline provides regulatory strategies of minimizing duplication of clinical data and requirement of bridging evidence for extrapolation of foreign clinical data to a new region.

The ICH E5 guideline suggests that a bridging study be conducted in the new region to generate additional information to bridge the foreign clinical data when the foreign clinical data contained in the Complete Clinical Data Package (CCDP) can not provide sufficient bridging evidence. By the ICH E5 guideline, a bridging study is therefore, defined as a supplementary study conducted in the new region to provide pharmacodynamic or clinical data on efficacy, safety, dosage, and dose regimen to allow extrapolation of the foreign clinical data to the population of the new region. In addition, the ICH E5 guideline also points out that the evaluation of the ability of extrapolation of the foreign clinical data relies upon the similarity of dose response, efficacy, and safety between the new and original regions either with or without dose adjustment. Several statistical procedures have been proposed to assess the similarity based on the additional information from the bridging study and the foreign clinical data in the CCDP see Shih (2001), Liu and Chow (2002), Chow et al. (2002), Liu et al. (2002, 2004), and Hsiao et al. (2003, 2005).

One of the crucial reasons for the ICH E5 guideline to emphasize on minimizing unnecessary duplication of generating clinical data in the new region is that sufficient information on efficacy, safety, dosage, and dose regimen has been already generated in the original region and is available in the CCDP. One should therefore borrow "strength" from the information on dose response, efficacy, and safety from the CCDP in the original region and incorporate them into the analysis of the additional data obtained from the bridging study. Liu et al. (2002) 
have proposed a Bayesian approach to synthesize the data generated by the bridging study and foreign clinical data generated in the original region for assessment of similarity based on superior efficacy of the test product over a placebo control. However, the results of the bridging studies using this approach will be overwhelmingly dominated by the results of the original region due to an imbalance of sample sizes between the regions. In other words, it is very difficult, if not impossible, to reverse the results observed in the original region even if the result of the bridging study is not consistent with those of the original region. However, this issue will occur for any methods for cross-study comparisons if the amount of information is seriously imbalanced between studies. Therefore, in this paper we propose a Bayesian approach with the use of a mixed prior information for assessment of similarity between the new and original region based on the concept of positive treatment effect. In Section 2, a Bayesian approach with a mixture prior information for assessment of similarity is suggested. The method for sample size determination is also given. A numerical example is presented in Section 3 to illustrate the Bayesian approach. Discussion and final remarks are given in Section 4.

\section{A MIXTURE PRIOR INFORMATION}

For simplicity, we only focus on the trials for comparing a test product and a placebo control. We consider the problem for assessment of similarity between the new and original region based on superior efficacy of the test product over a placebo control. Let $X_{i}$ and $Y_{j}$ be some efficacy responses for patients $i$ and $j$ receiving the test product and the placebo control, respectively, in the new region. For simplicity, both $X_{i}$ 's and $Y_{j}$ 's are normally distributed with variance $\sigma^{2}$. We assume that $\sigma^{2}$ is known, although it can generally be estimated. Let $\mu_{N T}$ and $\mu_{N P}$ be the population means of the test and placebo, respectively, and let $\Delta_{N}=\mu_{N T}-$ $\mu_{N P}$. The subscript $N$ in $\mu_{N T}, \mu_{N P}$, and $\Delta_{N}$ indicates the new region. Because the test product has been already approved in the original region due to its proven efficacy against placebo control, if the data collected from the bridging study in the new region also demonstrate a superior efficacy of the test pharmaceutical over placebo, then the efficacy observed in the population of the new region is claimed to be similar to that of the original region. This concept of similarity is referred to as the similarity by the positive treatment effect. In other words, this concept of similarity can be evaluated through the following hypothesis:

$$
H_{O}: \Delta_{N} \leq 0 \text { vs. } H_{A}: \Delta_{N}>0
$$

One, of course, can directly conduct a bridging study in the new region with similar sample size to the Phase III trials conducted in the original region to provide adequate power to test the above hypothesis for confirmation of the efficacy observed in the original region. However, tremendous resource will be allocated to conduct this type of the confirmation bridging trial in the new region. Even when the sponsor is willing to conduct such a trial, recruitment and length of the trial may be serious problems due to insufficient number of patients available in some small new regions. In addition, the valuable information about the efficacy, 
safety, and dosage contained in the CCDP are not fully utilized in the design and analysis of the bridging study. Furthermore, it is extremely critical to incorporate the information of the foreign clinical data into evaluation of the positive treatment effect for the bridging study conducted in the new region. Liu et al. (2002) proposed a Bayesian approach to use a normal prior to borrow strength from CCDP for evaluation of similarity between the new region and the original region. However, as mentioned before, their approach will be overwhelmingly dominated by the results of the original region if there is a serious imbalance in the information provided between the new and original regions.

Therefore, instead of the normal prior distribution used in Liu et al. (2002), we consider a mixture model for the prior information of $\Delta_{N}$. If no information from the CCDP generated in the original region is borrowed, the test for hypothesis (1) uses the information only generated by the bridging study conduced in the new region. This is equivalent to assuming a noninformative prior for $\Delta_{N}$. On the other hand, most of primary endpoints in a majority of therapeutic areas such hypertension, diabetes, depression, etc. follow or approximately follow a normal distribution. Therefore, it is quite reasonable to use a normal prior for summarization of the results in CCDP of the original region. As a result, the proposed mixture model of the prior information for $\Delta_{N}$ is a weighted average of two priors as given below

$$
\pi\left(\Delta_{N}\right)=\gamma \pi_{1}\left(\Delta_{N}\right)+(1-\gamma) \pi_{2}\left(\Delta_{N}\right)
$$

where $0 \leq \gamma \leq 1$. In $(2), \pi_{2}(\cdot)$ is a normal prior with mean $\theta_{0}$ and variance $\sigma_{0}^{2}$ summarizing the foreign clinical data about the treatment difference provided in the CCDP, whereas $\pi_{1}(\cdot)$ has three choices:

Case I: $\pi_{1}(\cdot) \equiv c$,

Case II: $\pi_{1}(\cdot)$ is a normal prior with mean 0 and variance $\sigma_{0}^{2}$,

Case III: $\pi_{1}(\cdot)$ is a normal prior with mean 0 and variance 1000 ,

In Case $\mathrm{I}, \pi_{1}(\cdot)$ is a noninformative prior, so $c$ can be any number. Our experience shows that changes in $c$ will not have any influence on the conclusion. Here, for simplicity, $c$ is set to be 1 . In Case II, $\pi_{1}$ is a normal prior with mean representing the null hypothesis: no treatment difference assumed for the prior of $\Delta_{N}$. In Case III, $\pi_{1}$ is a normal prior with vary large variance, 1000 , so it is very close to the noninformative prior considered in Case I. The proposed mixture model of the prior information for $\Delta_{N}$ in (2) indicates that a $\gamma$ value of 0 indicates that the prior $\pi$ is equivalent to the prior used in Liu et al. (2002), while $\gamma$ being 1 indicates that no strength of the evidence for the efficacy of the test product relative to placebo provided by the foreign clinical data in the CCDP from the original region would be borrowed. The choice of weight, $\gamma$, should reflect relative confidence of the regulatory authority on the evidence provided by the bridging study conducted in the new region vs. those provided by the original region. It should be determined by the regulatory authority of the new region by considering the difference in both intrinsic and extrinsic ethnical factors between the new and original regions.

Let $n_{T}$ and $n_{P}$ represent the numbers of patients studied for the test product and the placebo, respectively, in new region. Based on the clinical responses from 
the bridging study in new region, $\Delta_{N}$ can be estimated by

$$
\widehat{\Delta}_{N}=\bar{x}_{N}-\bar{y}_{N},
$$

where $\bar{x}_{N}=\sum_{i=1}^{n_{T}} x_{i} / n_{T}$ and $\bar{y}_{N}=\sum_{j=1}^{n_{P}} y_{j} / n_{P}$. For the choice of $\pi_{1}$ in Case I, the marginal density of $\widehat{\Delta}_{N}$ is

$$
m\left(\widehat{\Delta}_{N}\right)=\gamma+(1-\gamma) \frac{1}{\sqrt{2 \pi\left(\sigma_{0}^{2}+\tilde{\sigma}^{2}\right)}} \exp \left\{-\frac{\left(\widehat{\Delta}_{N}-\theta_{0}\right)^{2}}{2\left(\sigma_{0}^{2}+\tilde{\sigma}^{2}\right)}\right\},
$$

where $\tilde{\sigma}^{2}=\sigma^{2} / n_{T}+\sigma^{2} / n_{p}$. Given the bridging data and prior information, the posterior distribution of $\Delta_{N}$ is

$$
\begin{aligned}
\pi\left(\Delta_{N} \mid \widehat{\Delta}_{N}\right)=\frac{1}{m\left(\widehat{\Delta}_{N}\right)}\left\{\gamma \frac{1}{\sqrt{2 \pi} \tilde{\sigma}} \exp \left[-\frac{\left(\Delta_{N}-\widehat{\Delta}_{N}\right)^{2}}{2 \tilde{\sigma}^{2}}\right]\right. \\
\left.+(1-\gamma) \frac{1}{2 \pi \sigma_{0} \tilde{\sigma}} \exp \left[-\frac{\left(\Delta_{N}-\theta_{0}\right)^{2}}{2 \sigma_{0}^{2}}-\frac{\left(\Delta_{N}-\widehat{\Delta}_{N}\right)^{2}}{2 \tilde{\sigma}^{2}}\right]\right\}
\end{aligned}
$$

In both Case II and Case III, the marginal density of $\widehat{\Delta}_{N}$ is

$$
\begin{aligned}
m\left(\widehat{\Delta}_{N}\right)= & \gamma \frac{1}{\sqrt{2 \pi\left(\sigma_{1}^{2}+\tilde{\sigma}^{2}\right)}} \exp \left\{-\frac{\left(\widehat{\Delta}_{N}\right)^{2}}{2\left(\sigma_{1}^{2}+\tilde{\sigma}^{2}\right)}\right\} \\
& +(1-\gamma) \frac{1}{\sqrt{2 \pi\left(\sigma_{0}^{2}+\tilde{\sigma}^{2}\right)}} \exp \left\{-\frac{\left(\widehat{\Delta}_{N}-\theta_{0}\right)^{2}}{2\left(\sigma_{0}^{2}+\tilde{\sigma}^{2}\right)}\right\}
\end{aligned}
$$

where $\tilde{\sigma}^{2}=\sigma^{2} / n_{T}+\sigma^{2} / n_{P}$, and $\sigma_{1}^{2}=\sigma_{0}^{2}$ in Case II, and $\sigma_{1}^{2}=1000$ in Case III. Given the bridging data and prior information, the posterior distribution of $\Delta_{N}$ is

$$
\begin{aligned}
\pi\left(\Delta_{N} \mid \widehat{\Delta}_{N}\right)=\frac{1}{m\left(\widehat{\Delta}_{N}\right)} & \left\{\gamma \frac{1}{2 \pi \sqrt{\sigma_{1}^{2} \tilde{\sigma}^{2}}} \exp \left[-\frac{\left(\Delta_{N}\right)^{2}}{2 \sigma_{1}^{2}}-\frac{\left(\Delta_{N}-\widehat{\Delta}_{N}\right)^{2}}{2 \tilde{\sigma}^{2}}\right]\right. \\
& \left.+(1-\gamma) \frac{1}{2 \pi \sqrt{\sigma_{0}^{2} \tilde{\sigma}^{2}}} \exp \left[-\frac{\left(\Delta_{N}-\theta_{0}\right)^{2}}{2 \sigma_{0}^{2}}-\frac{\left(\Delta_{N}-\widehat{\Delta}_{N}\right)^{2}}{2 \tilde{\sigma}^{2}}\right]\right\}
\end{aligned}
$$

For each choice of $\pi_{1}$, given the data from the bridging study and prior information, similarity on efficacy in terms of a positive treatment effect for the new region can be concluded if the posterior probability of similarity

$$
\begin{aligned}
P_{S P} & =P\left(\mu_{N T}-\mu_{N P}>0 \mid \text { bridging data and prior }\right) \\
& =\int_{0}^{\infty} \pi\left(\Delta_{N} \mid \widehat{\Delta}_{N}\right) d \Delta_{N} \\
& >1-\alpha,
\end{aligned}
$$


for some prespecified $0<\alpha<0.5$. However, $\alpha$ is determined by the regulatory agency of the new region and should generally be smaller than 0.2 to ensure that posterior probability of similarity is at least $80 \%$.

\section{DETERMINATION OF SAMPLE SIZE}

Let $n_{N}$ represent the numbers of patients studied per treatment in the new region. We now describe the method of determination of sample size for the choice of $\pi_{1}$ in Case I. The generalization to other choices of $\pi_{1}$ can be derived similarly. Based on the discussion in the previous section, the marginal density of $\widehat{\Delta}_{N}$ in (3) can be re-expressed as

$$
m\left(\widehat{\Delta}_{N}\right)=\gamma+(1-\gamma) \frac{1}{\sqrt{2 \pi\left(\sigma_{0}^{2}+2 \sigma^{2} / n_{N}\right)}} \exp \left\{-\frac{\left(\widehat{\Delta}_{N}-\theta_{0}\right)^{2}}{2\left(\sigma_{0}^{2}+2 \sigma^{2} / n_{N}\right)}\right\} .
$$

The posterior distribution of $\Delta_{N}$, is therefore, given by

$$
\begin{aligned}
\pi\left(\Delta_{N} \mid \widehat{\Delta}_{N}\right)=\frac{1}{m(\widehat{\Delta})}\{\gamma & \frac{1}{\sqrt{4 \pi \sigma^{2} / n_{N}}} \exp \left[-\frac{\left(\Delta_{N}-\widehat{\Delta}_{N}\right)^{2}}{4 \sigma^{2} / n_{N}}\right] \\
& \left.+(1-\gamma) \frac{1}{2 \pi \sqrt{2 \sigma_{0}^{2} \sigma^{2} / n_{N}}} \exp \left[-\frac{\left(\Delta_{N}-\theta_{0}\right)^{2}}{2 \sigma_{0}^{2}}-\frac{\left(\Delta_{N}-\widehat{\Delta}_{N}\right)^{2}}{4 \sigma^{2} / n_{N}}\right]\right\} .
\end{aligned}
$$

Given $\alpha, \theta_{0}, \sigma_{0}^{2}, \sigma^{2}$ and the estimate $\widehat{\Delta}_{N}$, we can determine the sample size $n_{N}$ by finding the smallest $n_{N}$ such that the equation

$$
P_{S P}=\int_{0}^{\infty} \pi\left(\Delta_{N} \mid \widehat{\Delta}_{N}\right) d \Delta_{N}>1-\alpha
$$

is satisfied.

One approach to determination of $\Delta_{N}$ for sample size estimation of the bridging study is to adopt the "worst outcome criteria" approach suggested by Lawrence and Belisle (1997). Assume that $n_{O}$ represents the numbers of patients studied per treatment in the original region. The subscript $O$ in $n_{O}$ indicates the original region. We also assume that both efficacy endpoints of test drug and the placebo group in the original region have the same variance $\sigma^{2}$. Consequently, $\theta_{0}$ can be estimated by the difference in sample means of the original region and $\sigma_{0}^{2}=2 \sigma^{2} / n_{O}$ can be estimated by the pooled sample variance of mean difference. Hence, once $\theta_{0}$ and $\sigma_{0}^{2}$ are determined, $\sigma^{2}$ in (5) can be obtained by $n_{O} \sigma_{0}^{2} / 2$. Because the test product has been already approved in the original region due to its proven efficacy against placebo control, the ratio of $\theta_{0}$ to $\sigma_{0}$ is usually greater than 1.96 . Following the "worst outcome criteria" approach by Lawrence and Belisle (1997), the estimate of the treatment difference, $\widehat{\Delta}_{N}$, is chosen to be the lower bound of a 95\% confidence interval for $\Delta_{N}$ constructed from $\theta_{0}$ and $\sigma_{0}^{2}$. Table 1 provides the ratio of the sample size per treatment group for the bridging study to that of the CCDP $\left(n_{N} / n_{O}\right)$ for various combination of $\theta_{0}$ and variance $\sigma_{0}^{2}$ with $\alpha=0.1$ and $\alpha=0.2$.

From Table 1, the sample size required for the bridging study in the new region decreases as $\alpha$ or the ratio of $\theta_{0}$ to $\sigma_{0}^{2}$ increase and $\gamma$ decreases. Therefore, 
Table 1 The ratio of the sample size per treatment of the bridging study to that of the clinical trials in the CCDP at $\alpha=0.1$ or $\alpha=0.2$ for different combinations of $\theta_{0}$ and $\sigma_{0}^{2}$

\begin{tabular}{|c|c|c|c|c|c|c|c|c|}
\hline \multirow[b]{2}{*}{$\gamma$} & \multicolumn{2}{|c|}{$\theta_{0}=3, \sigma_{0}^{2}=1$} & \multicolumn{2}{|c|}{$\theta_{0}=4, \sigma_{0}^{2}=1$} & \multicolumn{2}{|c|}{$\theta_{0}=4, \sigma_{0}^{2}=2$} & \multicolumn{2}{|c|}{$\theta_{0}=5, \sigma_{0}^{2}=2$} \\
\hline & $\alpha=0.1$ & $\alpha=0.2$ & $\alpha=0.1$ & $\alpha=0.2$ & $\alpha=0.1$ & $\alpha=0.2$ & $\alpha=0.1$ & $\alpha=0.2$ \\
\hline 0 & $<.01$ & $<.01$ & $<.01$ & $<.01$ & $<.01$ & $<.01$ & $<.01$ & $<.01$ \\
\hline 0.1 & 0.63 & 0.09 & 0.17 & 0.04 & 1.29 & 0.20 & 0.34 & 0.09 \\
\hline 0.2 & 1.06 & 0.26 & 0.26 & 0.08 & 1.75 & 0.51 & 0.49 & 0.16 \\
\hline 0.3 & 1.24 & 0.39 & 0.31 & 0.11 & 1.92 & 0.68 & 0.56 & 0.20 \\
\hline 0.4 & 1.33 & 0.48 & 0.34 & 0.13 & 2.01 & 0.77 & 0.59 & 0.23 \\
\hline 0.5 & 1.39 & 0.53 & 0.35 & 0.14 & 2.06 & 0.82 & 0.61 & 0.25 \\
\hline 0.6 & 1.43 & 0.57 & 0.37 & 0.15 & 2.10 & 0.86 & 0.63 & 0.26 \\
\hline 0.7 & 1.46 & 0.60 & 0.38 & 0.16 & 2.13 & 0.89 & 0.64 & 0.27 \\
\hline 0.8 & 1.49 & 0.62 & 0.38 & 0.16 & 2.15 & 0.91 & 0.65 & 0.28 \\
\hline 0.9 & 1.50 & 0.64 & 0.39 & 0.17 & 2.17 & 0.93 & 0.66 & 0.28 \\
\hline \multirow[t]{2}{*}{1.0} & 1.52 & 0.65 & 0.39 & 0.17 & 2.18 & 0.94 & 0.66 & 0.29 \\
\hline & \multicolumn{2}{|c|}{$\theta_{0}=6, \sigma_{0}^{2}=2$} & \multicolumn{2}{|c|}{$\theta_{0}=6, \sigma_{0}^{2}=3$} & \multicolumn{2}{|c|}{$\theta_{0}=7, \sigma_{0}^{2}=3$} & \multicolumn{2}{|c|}{$\theta_{0}=8, \sigma_{0}^{2}=3$} \\
\hline$\gamma$ & $\alpha=0.1$ & $\alpha=0.2$ & $\alpha=0.1$ & $\alpha=0.2$ & $\alpha=0.1$ & $\alpha=0.2$ & $\alpha=0.1$ & $\alpha=0.2$ \\
\hline 0 & $<.01$ & $<.01$ & $<.01$ & $<.01$ & $<.01$ & $<.01$ & $<.01$ & $<.01$ \\
\hline 0.1 & 0.17 & 0.05 & 0.42 & 0.11 & 0.22 & 0.07 & 0.14 & 0.05 \\
\hline 0.2 & 0.24 & 0.08 & 0.57 & 0.19 & 0.30 & 0.11 & 0.18 & 0.07 \\
\hline 0.3 & 0.26 & 0.10 & 0.63 & 0.24 & 0.33 & 0.13 & 0.20 & 0.08 \\
\hline 0.4 & 0.28 & 0.11 & 0.66 & 0.26 & 0.35 & 0.14 & 0.21 & 0.09 \\
\hline 0.5 & 0.29 & 0.12 & 0.68 & 0.28 & 0.36 & 0.15 & 0.22 & 0.09 \\
\hline 0.6 & 0.30 & 0.12 & 0.70 & 0.29 & 0.36 & 0.15 & 0.22 & 0.09 \\
\hline 0.7 & 0.31 & 0.13 & 0.71 & 0.30 & 0.37 & 0.16 & 0.23 & 0.10 \\
\hline 0.8 & 0.31 & 0.13 & 0.72 & 0.30 & 0.37 & 0.16 & 0.23 & 0.10 \\
\hline 0.9 & 0.31 & 0.13 & 0.72 & 0.31 & 0.38 & 0.16 & 0.23 & 0.10 \\
\hline 1.0 & 0.32 & 0.14 & 0.73 & 0.31 & 0.38 & 0.16 & 0.23 & 0.10 \\
\hline
\end{tabular}

for a given value of $\theta_{0} / \sigma_{0}^{2}$, with proper selection of $\gamma$ and $\alpha$, reduction of the total sample size for the bridging study is possible when a statistically significant evidence of efficacy for the test product against placebo is provided in the original region. In particular, when all information from the original region is used, that is, when $\gamma=0$, the required sample size for the new region is always smaller than that of the original region. Also, the required sample size per treatment for the bridging study in the new region increases as $\gamma$ increases. For instance, when $\theta_{0}=4, \sigma_{0}^{2}=2$ (that is, two-side $p$-value $=0.0455$ for the original region), and $\alpha=0.2$, the sample size required per treatment for the bridging study increases from less than $1 \%$ of that required in the original region at $\gamma=0$ up to $94 \%$ at $\gamma=1.0$ with $\widehat{\Delta}_{N}=1.23$ (the lower bound of a $95 \%$ confidence interval for $\Delta_{N}$ constructed given that $\theta_{0}=4$ and $\sigma_{0}^{2}=2$ ). In other words, when less information borrowed from the original region is incorporated into the prior information, it would require a larger sample size of the bridging study in the new region.

\section{EXAMPLE}

Hypothetical datasets modified from our review experience of bridging studies are used to illustrate the proposed procedure. The CCDP provides the results of 
three randomized, placebo controlled trials for a new antidepressant (test drug) conducted in the original region. The design, inclusion, exclusion criteria, dose, and duration of these three trials are similar, and hence the three trials constituted as the pivotal trials for approval in the original region. The primary endpoint is the change from baseline of sitting diastolic blood pressure $(\mathrm{mmHg})$ at week 12 . Because the regulatory agency in the new region still has some concerns in ethnic differences, both intrinsically and extrinsically, a bridging study was conducted in the new region to compare the difference in efficacy between the new and original region. Three cases with various $\pi_{1}$, which is described in the previous section, are considered in this example. For each case, there are three scenarios to be considered. The first scenario presents the situation where no statistically significant difference in the primary endpoint exists between the test drug and placebo (twosided $p$-value $=0.6430$ ). The second situation is that the mean reduction of sitting diastolic blood pressure at week 12 of the test drug is statistically significantly greater than the placebo group (two-sided $p$-value $<0.0001$ ). The third scenario is the situation where due to the insufficient sample size of the bridging study, no statistical significance is found between the test drug and placebo although the magnitude of the difference between the test drug and placebo observed in the original region is preserved in the new region (two-sided $p$-value $=0.0716$ ). The number of patients and mean reduction and standard deviations of sitting diastolic blood pressure are provided in Table 2. The three scenarios are denoted as

Table 2 Descriptive statistics of reduction from baseline in sitting diastolic blood pressure $(\mathrm{mmHg})$

\begin{tabular}{|c|c|c|c|}
\hline \multirow[b]{2}{*}{ Region } & \multirow[b]{2}{*}{ Statistics } & \multicolumn{2}{|c|}{ Treatment group } \\
\hline & & Drug & Placebo \\
\hline \multirow[t]{3}{*}{ Original 1} & $\mathrm{~N}$ & 138 & 132 \\
\hline & Mean & -18 & -3 \\
\hline & Standard deviation & 11 & 12 \\
\hline \multirow[t]{3}{*}{ Original 2} & $\mathrm{~N}$ & 185 & 179 \\
\hline & Mean & -17 & -2 \\
\hline & Standard deviation & 10 & 11 \\
\hline \multirow[t]{3}{*}{ Original 3} & $\mathrm{~N}$ & 141 & 143 \\
\hline & Mean & -15 & -5 \\
\hline & Standard deviation & 13 & 14 \\
\hline \multirow{3}{*}{$\begin{array}{l}\text { New } 1 \\
\text { (Example 1) }\end{array}$} & $\mathrm{N}$ & 64 & 65 \\
\hline & Mean & -4.7 & -3.8 \\
\hline & Standard deviation & 11 & 11 \\
\hline New 2 & $\mathrm{~N}$ & 64 & 65 \\
\hline \multirow[t]{2}{*}{ (Example 2) } & Mean & -15 & -2 \\
\hline & Standard deviation & 11 & 11 \\
\hline New 3 & $\mathrm{~N}$ & 24 & 23 \\
\hline \multirow{2}{*}{ (Example 3) } & Mean & -11 & -4 \\
\hline & Standard deviation & 13 & 13 \\
\hline
\end{tabular}


New 1 (Example 1), New 2 (Example 2), and New 3 (Example 3), respectively. The alternative hypothesis of interest is that the difference in change from baseline in sitting diastolic blood pressure at week 12 between the test drug and placebo is less than 0 .

Using the technique of meta-analysis in Pettiti (2000) to integrate the results from both the original and new regions, we derive that $\theta_{0}=-13.91$ and $\sigma_{0}^{2}=0.59$. For the first two scenarios of the bridging studies considered here, $\hat{\sigma}^{2}=3.75$ for estimation of $\tilde{\sigma}^{2}$, while $\hat{\sigma}^{2}=14.39$ for estimation of $\tilde{\sigma}^{2}$ in the last scenario. Table 3 provides the values of $P_{S P}$ with various values of $\gamma$ for all three scenarios and all three choices of $\pi_{1}$.

For Example 1, the difference in mean reduction of sitting blood pressure between the test drug and placebo is $0.9 \mathrm{mmHg}$ which is strikingly different from those obtained from three trials conducted in the original region. If the regulatory agency allows all information of the original region to be used for evaluation of similarity between the new and original region, $\gamma$ is set to be 0 and hence $P_{S P} \approx$ 1.00 regardless of the choice of $\pi_{1}$. That is the same result obtained by Liu et al. (2002). Therefore, we conclude that the efficacy observed in the bridging study of the new region is similar to the efficacy from the original region even if there is no statistically significant difference in the primary endpoint between the test drug and placebo. This phenomenon implies that when all information from the original region is used, the results of the bridging studies will be overwhelmingly dominated by those of the original region. On the other hand, if $\gamma \geq 0.1$, then $P_{S P}$ always drops to around 0.6789 in Case I, 0.5680 in Case II, and 0.6786 in Case III. Accordingly, we can not conclude that the results of the new region are similar to those of the original region if $1-\alpha$ is set to be greater than $80 \%$. More specifically, $P_{S P}$ drops to 0.8 when $\gamma=1.0 E-0.8$ in Case I, $\gamma=9.0 E-08$ in Case II, and $\gamma=8.0 E-0.7$ in Case III. That is, even with use of very little information from the new region, our proposed procedure reaches a conclusion that is more consistent with the evidence provided by the new region.

Table 3 Values of $P_{S P}$ derived from examples 1, 2, and 3 with various values of $\gamma$

\begin{tabular}{|c|c|c|c|c|c|c|c|c|c|}
\hline \multirow[b]{3}{*}{$\gamma$} & \multicolumn{9}{|c|}{$P_{S P}$} \\
\hline & \multicolumn{3}{|c|}{ Example 1} & \multicolumn{3}{|c|}{ Example 2} & \multicolumn{3}{|c|}{ Example 3} \\
\hline & Case I & Case II & Case III & Case I & Case II & Case III & Case I & Case II & Case III \\
\hline 0.0 & $\approx 1$ & $\approx 1$ & $\approx 1$ & $\approx 1$ & $\approx 1$ & $\approx 1$ & $\approx 1$ & $\approx 1$ & $\approx 1$ \\
\hline 0.1 & 0.6789 & 0.5680 & 0.6786 & 0.9999 & $\approx 1$ & $\approx 1$ & 0.9727 & 0.9656 & 0.9980 \\
\hline 0.2 & 0.6789 & 0.5680 & 0.6786 & 0.9999 & $\approx 1$ & $\approx 1$ & 0.9700 & 0.9309 & 0.9957 \\
\hline 0.3 & 0.6789 & 0.5680 & 0.6786 & 0.9999 & $\approx 1$ & $\approx 1$ & 0.9690 & 0.8960 & 0.9933 \\
\hline 0.4 & 0.6789 & 0.5680 & 0.6786 & 0.9999 & $\approx 1$ & $\approx 1$ & 0.9685 & 0.8607 & 0.9906 \\
\hline 0.5 & 0.6789 & 0.5680 & 0.6786 & 0.9999 & $\approx 1$ & $\approx 1$ & 0.9682 & 0.8252 & 0.9877 \\
\hline 0.6 & 0.6789 & 0.5680 & 0.6786 & 0.9999 & $\approx 1$ & $\approx 1$ & 0.9680 & 0.7893 & 0.9844 \\
\hline 0.7 & 0.6789 & 0.5680 & 0.6786 & 0.9999 & $\approx 1$ & $\approx 1$ & 0.9678 & 0.7532 & 0.9807 \\
\hline 0.8 & 0.6789 & 0.5680 & 0.6786 & 0.9999 & $\approx 1$ & $\approx 1$ & 0.9677 & 0.7167 & 0.9766 \\
\hline 0.9 & 0.6789 & 0.5680 & 0.6786 & 0.9999 & $\approx 1$ & $\approx 1$ & 0.9676 & 0.6800 & 0.9719 \\
\hline 1.0 & 0.6789 & 0.5680 & 0.6786 & 0.9999 & 0.9934 & $\approx 1$ & 0.9675 & 0.6429 & 0.9665 \\
\hline
\end{tabular}


For Example 2, the difference in mean reduction of sitting blood pressure between the test drug and placebo is $13 \mathrm{mmHg}$ which is quite consistent with those obtained from three trials conducted in the original region. As expected, the values of $P_{S P}$ in Example 2 appear to be close to 1.00 regardless of the choice of $\gamma$ and $\pi_{1}$. We can, therefore, conclude similarity between the new and original regions. Again, our procedure obtains a conclusion that is consistent with the evidence provided by the results of the bridging study conducted in the new region.

For Example 3, the magnitude of the mean difference is 7, is that, the similar situation in the original region. However, the difference is not statistically significant at the 5\% level due to the smaller sample size and the larger variability in the new region. As seen from Table 3, the values of $P_{S P}$ are all greater than 0.9665 for all values of $\gamma$ between 0 and 1 when $\pi_{1}$ is chosen as in Case I and Case III. Hence, similarity between the new and original regions is concluded if $\alpha$ is less than $10 \%$. With the strength of the substantial evidence of efficacy borrowed from the CCDP of the original region, our procedure can prove the similarity of efficacy between the new and the original region when a nonsignificant efficacy result but with a similar magnitude is observed in the bridging study. When $\pi_{1}$ is chosen as in Case II, the values of $P_{S P}$ gradually decrease from around 1 to 0.6429 when the values of $\gamma$ increase from 0 to 1 . In particular, $P_{S P}$ drops to be less than 0.80 if $\gamma>0.50$. Therefore, we cannot conclude that the results of the new region are similar to those of the original region if $1-\alpha$ is set to be greater than $80 \%$. As indicated in Case II, $\pi_{1}$ is a normal prior which represents the null hypothesis: no treatment difference assumed for the prior of $\Delta_{N}$. Therefore, as $\gamma$ increases, influence of the prior $\pi$ becomes stronger towards the null hypothesis of no treatment difference. In this case, care should be exercised to choose $\gamma$.

This example demonstrates that with proper selection of $\gamma$ by the regulatory agency of the new region, our Bayesian approach with the mixture prior in (2) reaches a conclusion which is much more in line with the results of the bridging study in the new region. In addition, our approach can avoid the difficulty arising from imbalance amount of information between two regions, an issue suffered by the Bayesian procedure proposed by Liu et al. (2002).

For sample size determination, to be conservative, we use the "worst outcome criteria" approach by Lawrence and Belisle (1997) with $\theta_{0}=-8, \sigma_{0}^{2}=2.56$, and $\sigma^{2}=14.39$. Hence, the lower limit of the $95 \%$ confidence interval is -11.136 . When $\alpha=0.1$, the required sample size for the bridging study in the new region is 26 per treatment group for $\gamma=0$. The required sample size per group decreases to 11 if $\alpha=0.1$ and $\gamma>0$.

\section{DISCUSSION AND FINAL REMARKS}

Herein, a Bayesian method with a mixture prior information has been suggested to synthesize the data from both the bridging study and the original region for assessment of bridging evidence. The proposed prior information is a weighted average of a noninformative prior and a normal prior, or two normal priors. With an appropriate choice of weight $\gamma$, the evaluation of similarity based on the integrated results of the bridging studies in the new region and those from the original region will no longer be overwhelmingly dominated by the results of the original region due to an imbalance of sample sizes between the regions. Therefore, 
the proposed procedure can avoid the situation of concluding similarity between the new and original region when the efficacy result of the test drug observed the bridging study of the new region is same as or even worse than that of the placebo group. However, as demonstrated in Example, similarity between the new and original region will be concluded when the difference in primary endpoint between the test drug and placebo observed in the bridging study is of the same magnitude of that obtained from the original region although it is not statistically significant due to the small sample size of the bridging study. As a result, our proposed procedure not only can reach a conclusion that is more consistent with the results obtained from the bridging study but also can achieve the objective of minimizing duplication of clinical evaluation in the new region as specified in the ICH E5 guidance.

Selection of weight $\gamma$ by the regulatory agency in the new region should consider all differences in both intrinsic and extrinsic ethnical factors between the new and original regions and at the same time should also reflect their belief on the evidence of efficacy provided in the CCDP of the original region. As mentioned before, a bridging study is conducted in the new region because of concerns on ethnic differences between the new and original regions, therefore, it is suggested that weight $\gamma$ be greater than 0 . However, from Table 1 and examples given in Section 4 , the weight has a very minimal effect on the sample size of the bridging study and $P_{S P}$ once it is greater than 0.2. In Case I, the prior is $\pi\left(\Delta_{N}\right)=\gamma c+(1-\gamma)$ $\pi_{2}\left(\Delta_{N}\right)$. The prior is non-informative because

$$
\int \pi\left(\Delta_{N}\right) d \Delta_{N}=\infty \text { for any } c .
$$

We have calculated $P_{S P}$ under different values of $c$ for Examples 1 and 3. The results are shown in Tables 4 and 5. As it can be seen from Tables 4 and 5, the changes in $c$ did not have strong influence on the values of $P_{S P}$.

It should be noted, however, that we use a normal prior for summarization of the results in CCDP of the original region as described above. Nonetheless, it might be possible to use other prior distributions to borrow strength from CCDP. To see

Table 4 Values of $\gamma$ corresponding to various values of $P_{S P}$ in Example 1 under different values of $c$

\begin{tabular}{lccccc}
\hline \multicolumn{5}{c}{ Case I in Example 1} \\
$\gamma$ & $c=1$ & $c=5$ & $c=10$ & $c=20$ & $c=50$ \\
\cline { 2 - 6 } 0.0 & $\approx 1$ & $\approx 1$ & $\approx 1$ & $\approx 1$ & $\approx 1$ \\
0.1 & 0.6789 & 0.6789 & 0.6789 & 0.6789 & 0.6789 \\
0.2 & 0.6789 & 0.6789 & 0.6789 & 0.6789 & 0.6789 \\
0.3 & 0.6789 & 0.6789 & 0.6789 & 0.6789 & 0.6789 \\
0.4 & 0.6789 & 0.6789 & 0.6789 & 0.6789 & 0.6789 \\
0.5 & 0.6789 & 0.6789 & 0.6789 & 0.6789 & 0.6789 \\
0.6 & 0.6789 & 0.6789 & 0.6789 & 0.6789 & 0.6789 \\
0.7 & 0.6789 & 0.6789 & 0.6789 & 0.6789 & 0.6789 \\
0.8 & 0.6789 & 0.6789 & 0.6789 & 0.6789 & 0.6789 \\
0.9 & 0.6789 & 0.6789 & 0.6789 & 0.6789 & 0.6789 \\
1.0 & 0.6789 & 0.6789 & 0.6789 & 0.6789 & 0.6789 \\
\hline
\end{tabular}


Table 5 Values of $\gamma$ corresponding to various values of $P_{S P}$ in Example 3 under different values of $c$

\begin{tabular}{lccccc}
\hline \multicolumn{5}{c}{ Case I in Example 3} \\
$\gamma$ & $c=1$ & $c=5$ & $c=10$ & $c=20$ & $c=50$ \\
\cline { 2 - 6 } 0.0 & $\approx 1$ & $\approx 1$ & $\approx 1$ & $\approx 1$ & $\approx 1$ \\
0.1 & 0.9727 & 0.9676 & 0.9681 & 0.9678 & 0.9676 \\
0.2 & 0.9700 & 0.9676 & 0.9678 & 0.9676 & 0.9676 \\
0.3 & 0.9690 & 0.9675 & 0.9677 & 0.9676 & 0.9675 \\
0.4 & 0.9685 & 0.9675 & 0.9676 & 0.9676 & 0.9675 \\
0.5 & 0.9682 & 0.9675 & 0.9676 & 0.9675 & 0.9675 \\
0.6 & 0.9680 & 0.9675 & 0.9675 & 0.9675 & 0.9675 \\
0.7 & 0.9678 & 0.9675 & 0.9675 & 0.9675 & 0.9675 \\
0.8 & 0.9677 & 0.9675 & 0.9675 & 0.9675 & 0.9675 \\
0.9 & 0.9676 & 0.9675 & 0.9675 & 0.9675 & 0.9675 \\
1.0 & 0.9675 & 0.9675 & 0.9675 & 0.9675 & 0.9675 \\
\hline
\end{tabular}

whether changes in the prior distribution cause any significant impact in conclusion, we repeat calculations of $P_{S P}$ for these three examples shown in the preceding section using two other prior distributions for $\pi_{2}$ by choosing $\pi_{1}$ as in Case I. The first one is the double exponential distribution (a symmetrical distribution), and the other is the lognormal distribution (a skewed distribution). For the double exponential distribution, $P_{S P}=0.9878$ for $\gamma=0$, and are close to 0.6789 for other choices of $\gamma$ in Example 1. Also all the values of $P_{S P}$ are greater than 0.9675 for all $\gamma$ in both Examples 2 and 3 with the double exponential prior. With respect to the lognormal distribution, the value of $P_{S P}$ is equal to 1.00 for $\gamma=0$, is close to 0.6789 for other choices of $\gamma$ in Example 1. Again, with the lognormal prior, all $P_{S P}$ 's are greater than 0.9675 for all values of $\gamma$ in both Examples 2 and 3. In summary, three different distributions used for $\pi_{2}$ reach the same conclusion. Therefore our proposed procedure is quite robust to choice of different prior distributions. On the other hand, the error rate of decision-making on similarity by Bayesian methods also requires further research.

\section{REFERENCES}

Chow, S. C., Shao, J., Hu, O. Y. P. (2002). Assessing sensitivity and similarity in bridging studies. Journal of Biopharmaceutical Statistics 12:385-400.

Hsiao, C. F., Xu, J. Z., Liu, J. P. (2003). A group sequential approach to evaluation of bridging studies. Journal of Biopharmaceutical Statistics 13:793-801.

Hsiao, C. F., Xu, J. Z., Liu, J. P. (2005). A two-stage design for bridging studies. Journal of Biopharmaceutical Statistics 15:75-83.

ICH, International Conference on Harmonisation. (1998). Tripartite guidance E5 ethnic factors in the acceptability of foreign data. The U.S. Federal Register 83:31790-31796.

Lawrence, J., Belisle, P. (1997). Bayesian sample size determination for normal means and differences between normal means. The Statistician 46:209-226.

Liu, J. P., Chow, S. C. (2002). Bridging studies for clinical development. Journal of Biopharmaceutical Statistics 12:357-369.

Liu, J. P., Hsiao, C. F., Hsueh, H. M. (2002). Bayesian approach to evaluation of bridging studies. Journal of Biopharmaceutical Statistics 12:401-408. 
Liu, J. P., Hsueh, H. M., Chen, J. J. (2002). Sample size requirement for evaluation of bridging evidence. Biometrical Journal 44:969-981.

Liu, J. P., Hsueh, H. M., Hsiao, C. F. (2004). Bayesian non-inferior approach to evaluation of bridging studies. Journal of Biopharmaceutical Statistics 14:291-300.

Pettiti, D. B. (2000). Meta-Analysis, Decision Analysis, and Cost-Effectiveness Analysis. New York: Oxford University Press, pp. 90-129.

Shih, W. J. (2001). Clinical trials for drug registration in Asian-Pacific countries: proposal for a new paradigm from a statistical perspective. Controlled Clinical Trials 22:357-366. 
Copyright of Journal of Biopharmaceutical Statistics is the property of Taylor \& Francis Ltd and its content may not be copied or emailed to multiple sites or posted to a listserv without the copyright holder's express written permission. However, users may print, download, or email articles for individual use. 